\title{
SINDROME METABÓLICO EN MUJERES DIABETICAS TIPO 2 SEGÚN LA FEDERACION INTERNACIONAL DE DIABETES Y ATP-IIIa
}

\section{Metabolic syndrome in women with type 2 diabetes mellitus by international diabetes federation criteria and ATP-IIla}

\author{
* Ligia Aracely Aguirre Moreno, *Junior Iván Torres Castillo, *Cheryl Giselle Núñez Madrid.
}

\section{RESUMEN}

El síndrome metabólico se define como un conjunto de factores de riesgo cardiovascular y diabetes mellitus tipo II. Estos factores: obesidad abdominal, hiperglicemia o resistencia a la insulina, dislipidemia e hipertensión arterial en un mismo individuo. Objetivo: Comparar la frecuencia de Síndrome Metabólico en mujeres adultas con diabetes mellitus tipo 2 según los criterios de la Federación Internacional de Diabetes (IDF) y del Programa Nacional de Colesterol- ATP III modificada (NCEP-ATPIIla). Material y métodos: Estudio cuantitativo, alcance descriptivo correlacional, de corte transversal en el que se incluyeron 102 mujeres con diabetes mellitus tipo 2 , no embarazadas, mayores de 18 años usuarios del Centro de salud FESITRANH del departamento de Cortés (50 pacientes), y del Centro de salud de Zacapa, Santa Bárbara (52 pacientes). Se realizó una historia clínica detallada y se llevaron a cabo las medidas antropométricas y de laboratorio para determinar el Síndrome Metabólico con ambos criterios. El Síndrome Metabólico se definió de acuerdo a los criterios de la IDF y de la NCEP-ATPIIla. Resultados: Las frecuencias del Diagnóstico del síndrome metabólico con los diferentes criterios fueron de $86.3 \%$ y $55.9 \%$ para ATP-IIla e IDF respectivamente con un chi cuadrado de 0.919 . El diagnostico por área geográfica según criterios de IDF fue de $50.9 \%$ con chi cuadrado 0.987, y con ATP-IIla del $55.7 \%$ con un chi cuadrado de 0.017 . Conclusión: La frecuencia del síndrome metabólico definida por los criterios de la ATP-IIla fue mayor que utilizando los criterios de IDF por área geográfica.

*Doctor en medicina y cirugía. Egresado de la Universidad Católica de Honduras, campus San Pedro Sula.

Dirigir correspondencia a: cheryl_nunez@hotmail.com

Recibido: 25 de mayo $2016 \quad$ Aprobado: 28 de noviembre 2016

\section{PALABRAS CLAVE}

Circunferencia de la cintura, Diabetes Mellitus, Honduras, Mujeres.

\section{ABSTRACT}

Metabolic syndrome is defined as a set of cardiovascular risk factors and type II diabetes mellitus, including: abdominal obesity, hyperglycemia or insulin resistance, dyslipidemia and high blood pressure in the same individual. Objective: Compare the frequency of metabolic syndrome in adult females with type II diabetes mellitus according to International Diabetes Federation (IDF) criteria and the modified National Cholesterol-ATP III Program (NCEP-ATPIIla). Material and methods: A cross-sectional, descriptive, study was carried out in which 102 women with type II diabetes mellitus, not pregnant, aged 18 and over were enrolled in the FESITRANH health center in the department of Cortes (50 patients) and of Zacapa's Health Center, Santa Bárbara (52 patients). A detailed clinical history was made, anthropometric and laboratory measurements were performed to determine the Metabolic Syndrome with both criteria. The metabolic syndrome was defined according to the criteria of the IDF and the NCEP-ATPIIla. Results: The frequency of diagnosis of metabolic syndrome with the different criteria were $86.3 \%$ and $55.9 \%$ for ATP-IIIa and IDF respectively with a chi-square of 0.919 . Diagnosis by geographical area according to IDF criteria was $50.9 \%$ with chi square 0.987 , and with ATP-IIla of $55.7 \%$ with a chi square of 0.017 . Conclusion: The frequency of the metabolic syndrome defined by the ATP-IIla criteria was higher than using the IDF criteria by geographical area. 


\section{KEYWORDS}

Waist circumference, Mellitus Diabetes, Women, Honduras.

\section{INTRODUCCION}

El aumento progresivo de Diabetes Mellitus, Obesidad y Enfermedad Cardiovascular se ha convertido en un importante problema de salud pública en todo el mundo, ${ }^{(1)}$ los países latinoamericanos enfrentan una epidemia de enfermedades crónicas que han sido la principal causa de muerte en los últimos años; ${ }^{(2)}$ la combinación de éstas patologías es un problema de salud que compete a nivel mundial, el concepto de diabetes no debe abordarse como una patología aislada, ya que es parte de un complejo de enfermedades más extenso: el síndrome metabólico. ${ }^{(3)}$

El síndrome metabólico se define como un conjunto de factores de riesgo cardiovascular, y diabetes mellitus tipo II; entre estos factores: obesidad abdominal, hiperglicemia o resistencia a la insulina, dislipidemia e hipertensión arterial en un mismo individuo, ${ }^{(4)}$ además de estos factores se han asociado la hipercoagulabilidad, inflamación y disfunción endotelial. ${ }^{(5)}$

La historia del síndrome metabólico es amplia, data desde hace 250 años, con diversas investigaciones de médicos científicos. ${ }^{(6)}$ Sin embargo, la primera definición oficial aparece en 1998 elaborada por la Organización Mundial de la Salud (OMS), ${ }^{(7)}$ posteriormente han surgido varias propuestas como The Third Report National Cholesterol Education Program (NCEP-ATP III), ${ }^{(8)}$ del European Group for the Study of Insulin Resistance ${ }^{(9)}$ y la mas actual, de International Diabetes Federation (IDF). ${ }^{(10)}$ En Honduras los datos acerca de la prevalencia del síndrome metabólico en la población diabética son escasos, existen dos estudios realizados sobre este tema uno de ellos en El Progreso Yoro, aplicado a pacientes hipertensos, en el que se obtuvo una frecuencia de $70.5 \%$ de síndrome metabólico, ${ }^{(11)}$ y el segundo estudio realizado en Tegucigalpa, en el Hospital Escuela aplicado a pacientes diabéticos e hipertensos con una frecuencia de $98 \%$ para los diabéticos-hipertensos, $60 \%$ en los diabéticos y $62.5 \%$ en los hipertensos según los criterios del ATP III. ${ }^{(12)}$ Es importante poder determinar la frecuencia del síndrome metabólico en las pacientes mujeres diabéticas, pues de esta forma es posible identificar a aquellas que presentan mayor riesgo cardiovascular, ya que el perfil de dicho riesgo es diferente para aquellas pacientes diabéticos que presenten o no el síndrome metabólico. ${ }^{(3)}$

El riesgo de enfermedades cardiovasculares aumenta 2.5 veces más en aquellos pacientes diabéticos que presentan el síndrome metabólico y, 1.5 veces más el riesgo de mortalidad. ${ }^{(13)}$

El objetivo del presente estudio fue comparar la frecuencia de Síndrome Metabólico en femeninas adultas con diabetes mellitus tipo 2 según los criterios de la Federación Internacional de Diabetes (IDF) del Programa Nacional de Colesterol-ATP III modificada (NCEP-ATPIIla) mayores de 18 años usuarios del Centro de salud FESITRANH de la ciudad de San Pedro Sula, Cortés y del Centro de salud de Zacapa, Santa Bárbara, de enero a noviembre del 2014.

\section{PACIENTES Y METODOS}

Estudio con enfoque cuantitativo, diseño transversal y alcance descriptivo correlacional. Se comparó la frecuencia de Síndrome Metabólico en femeninas adultas con diabetes mellitus tipo 2 según los criterios de la Federación Internacional de Diabetes (IDF) y del Programa Nacional de Colesterol- ATP III modificada (NCEP-ATPIIla) en mayores de 18 años usuarias del Centro de Salud FESITRAN de la ciudad de San Pedro Sula, Cortés y del Centro de salud de Zacapa, Santa Bárbara, de enero a noviembre del 2014.

Se realizó un muestreo no probabilístico por conveniencia, incluyendo 102 mujeres (50 pacientes del CESAMO FESITRANH y 52 del CESAMO de Zacapa) con diabetes mellitus tipo 2 , no embarazadas, mayores de 18 años y que brindaron su consentimiento informado. Se excluyeron: mujeres embarazadas, a quienes 
padecieran enfermedades físicas o mentales invalidantes, tratamiento con esteroides y portadores de patología tiroidea descompensada, pacientes que no completaron los exámenes laboratoriales necesarios para realizar el estudio y a quienes no brindaron su consentimiento.

El instrumento para la recolección de datos fue adecuado de una encuesta obtenida de la versión panamericana de STEPS ${ }^{(14)}$ para vigilancia de los factores de riesgo de las enfermedades crónicas, en asociación con la Organización Mundial de la Salud y la Organización Panamericana de la Salud. Previo a su ejecución fue evaluado y aprobado por una comisión médica local. Se aplicó una prueba piloto con el fin de determinar si era aplicable para la población en cuestión. Dicho cuestionario fue llenado por el encuestador previamente entrenado.

La presencia de Síndrome Metabólico fue definida de acuerdo con los criterios de la IDF y de la NCEP-ATP-III modificada (NCEP-ATPIIla) (Ver Tabla No. 1.)

Tabla No. 1: Criterios diagnósticos de Síndrome Metabólico

\begin{tabular}{|c|c|c|}
\hline & ATP-IIIa & IDF \\
\hline $\mathrm{TA}(\mathrm{mmHg})$ & $>130 / 85$ & $\begin{array}{l}>130 / 85 \text { o un tra- } \\
\text { ta miento contra } \\
\text { la hipertensión } \\
\text { diagnósticada } \\
\text { anteriormente }\end{array}$ \\
\hline $\begin{array}{l}\text { GPA (mg/dl) } \\
\text { C-HDL (mg/dl) }\end{array}$ & $>100$ & $>100$ \\
\hline - Hombres & $<40$ & $<40$ \\
\hline - Mujeres & $<50$ & $<50$ \\
\hline TG (mg/dL) & $>150$ & $>150$ \\
\hline $\begin{array}{c}\text { Cintura }(\mathrm{cm}) \\
\text { - Hombres } \\
\text { - Mujeres }\end{array}$ & $\begin{array}{l}>102 \\
>88\end{array}$ & $\begin{array}{l}>90 \\
>80\end{array}$ \\
\hline
\end{tabular}

Fuente: Crepaldi, Gaetano and SM. El síndrome metabólico contexto historico. Federación Internacional de Diabetes. Diabetes voice. 2006;51: 9-13. ${ }^{(6)}$ Definición ATPIIla: Presencia de tres o más de los criterios especificados en el cuadro. Definición de IDF: Presencia de obesidad abdominal definida por una circunferencia abdominal $>90 \mathrm{~cm}$ en hombre $y>80 \mathrm{~cm}$ en la mujer por el grupo étnico y más dos de los criterios anotados en el cuadro.

Abreviaturas: GPA = Glucosa plasmática en ayuno. $T A=$ Tensión arterial

$\mathrm{C}-\mathrm{HDL}=$ Colesterol de lipoproteínas de alta densidad $\mathrm{TG}=$ Triglicéridos .

IC/C = Índice cintura-cadera. ATPIII = Panel de Tratamiento del Colesterol en Adultos. IDF = Federación Internacional de Diabetes.
Posterior a la recolección y análisis de los datos obtenidos se utilizó el programa estadístico SPSS 18, para para el análisis de los resultados. Se calculó chi2 para correlación entre las variables.

\section{RESULTADOS}

En el estudio participaron un total de 102 sujetos del sexo femenino, con una edad media de $57 \pm 12$ años. La edad más frecuente fue 64 años, la de menor edad tenía 20 y la edad máxima fue de 84 años. La media de los años de escolaridad fue de 2.3 , oscilando entre $1 \mathrm{y}$ 7 años de estudios.

Utilizando los criterios de la ATP III, la frecuencia del síndrome metabólico fue de $86.3 \%$ y con los criterios de la IDF fue de $55.9 \%$. Al analizar estadísticamente la relación existente entre los criterios de Síndrome Metabólico se demostró que no existe diferencias significativas entre la definición de IDF y la definición de ATPIII (chi cuadrado 0.9, p>0.05), (Ver tabla No. 2.)

Tabla No. 2: Frecuencia de diagnóstico de Síndrome Metabólico según ATPIII/IDF

\begin{tabular}{|c|c|c|c|c|}
\hline \multirow{5}{*}{$\begin{array}{c}\text { Diagnóstico } \\
\text { de } \\
\text { Síndrome } \\
\text { Metabólico }\end{array}$} & & $\begin{array}{l}\text { SEGÚN } \\
\text { ATP-III }\end{array}$ & $\begin{array}{l}\text { SEGÚN } \\
\text { IDF }\end{array}$ & $\begin{array}{l}\text { CHI CUA- } \\
\text { DRADO }\end{array}$ \\
\hline & & Frecuencia & Frecuencia & \multirow{4}{*}{0.919} \\
\hline & SI & $88(86.3 \%)$ & $57(55.9 \%)$ & \\
\hline & NO & $14(13.7 \%)$ & $45(44.1 \%)$ & \\
\hline & & $102(100 \%)$ & $102(100 \%)$ & \\
\hline
\end{tabular}

Fuente: instrumento de recolección de datos.

Se determinó la frecuencia del síndrome estudiado ajustado a edad, obteniendo que de las pacientes con síndrome metabólico según criterios de ATP-IIla, 39.8\% tenían edades comprendidas entre 45 a 59 años, y según criterios de IDF $38.6 \%$ de las pacientes de ese mismo grupo etario, tenían dicho síndrome. (Ver tabla No. 3.) 
Tabla No. 3: Frecuencia de Síndrome Metabólico según ATPIII/IDF por edad

\begin{tabular}{|l|lll|l|l|}
\hline RANGO DE EDAD & \multicolumn{1}{|c|}{ ATP si } & \multicolumn{1}{c|}{ ATP no } & & \multicolumn{1}{c|}{ IDF si } & \multicolumn{1}{c|}{ IDF no } \\
\hline 18 a 29 & $4(4.5 \%)$ & 0 & $2(3.5 \%)$ & $2(4.4 \%)$ \\
\hline 30 a 34 & $15(17.1 \%)$ & 0 & $6(10.5 \%)$ & $9(20 \%)$ \\
\hline 45 a 59 & $35(39.8 \%)$ & $6(42.8)$ & $22(38.6 \%)$ & $19(42.2 \%)$ \\
\hline 60 y mas & $34(38.6 \%)$ & $8(57.2 \%)$ & $27(47.4 \%)$ & $15(33.4 \%)$ \\
\hline Total & $88(100 \%)$ & $14(100 \%)$ & $57(100 \%)$ & $45(100 \%)$ \\
\hline
\end{tabular}

Fuente: instrumento de recolección de datos.

Además se analizó la frecuencia del síndrome metabólico según el área geográfica estudiada, de un total de 102 pacientes 50 pertenecían al CESAMO de Zacapa y 52 al CESAMO de Fesitrah. Obteniéndose una frecuencia de $44.3 \%$ para las pacientes de CESAMO Fesitrahn y $55.7 \%$ para las pacientes de CESAMO Zacapa según ATP-IIla. Y $49.1 \%$ en las pacientes del CESAMO Fesitranh, $50.9 \%$ en las pacientes de CESAMO Zacapa según IDF. (Ver tabla No. 4.)
Al analizar estadísticamente la relación existente entre el área geográfica en estudio y los criterios de Síndrome Metabólico se demostró que no existe diferencias significativas entre área geográfica y la definición de IDF ya que se obtuvo chi cuadrado $0.987, p>0.05$, sin embargo, con los criterios ATP-Illa se encontró que existe relación entre ambas variables obteniendo un chi cuadrado $0.017, p<0.05$.

Tabla No. 4: Relación del Síndrome Metabólico y lugar de procedencia

\begin{tabular}{|c|c|c|c|c|c|c|}
\hline $\begin{array}{c}\text { Síndrome Metabólico } \\
\text { Criterios: }\end{array}$ & \multicolumn{2}{|c|}{ ATP-IIIa } & \multirow[t]{2}{*}{ Estadistico } & \multicolumn{2}{|c|}{ IDF } & Estadístico \\
\hline Lugar de procedencia & Si & No & & Si & No & \multirow{4}{*}{$\begin{array}{c}\text { Chi2 } \\
\text { cuadrado } \\
0.987\end{array}$} \\
\hline $\begin{array}{l}\text { CESAMO } \\
\text { ZACAPA }\end{array}$ & $49(55.7 \%)$ & $3(21.4 \%)$ & \multirow{3}{*}{$\begin{array}{c}\text { Chi2 } \\
\text { cuadrado } \\
0.017\end{array}$} & $29(50.9 \%)$ & $\begin{array}{c}23 \\
(51.1 \%)\end{array}$ & \\
\hline $\begin{array}{l}\text { CESAMO } \\
\text { FESITRANH }\end{array}$ & $39(44.3 \%)$ & $11(78.6 \%)$ & & $28(49.1 \%)$ & $\begin{array}{c}22 \\
(48.9 \%) \\
\end{array}$ & \\
\hline Total & $88(100 \%)$ & $14(100 \%)$ & & $57(100 \%)$ & $45(100 \%)$ & \\
\hline
\end{tabular}

Fuente: instrumento de recolección de datos.

\section{DISCUSION}

Se ha evidenciado en las últimas actualizaciones la importancia del diagnóstico de síndrome metabólico debido al riesgo cardiovascular que este representa, en este caso en particular para las pacientes diabéticas. Sin embargo, a pesar de las diversas publicaciones sobre el tema y los diferentes criterios utiliza- dos para diagnosticarlo no se han logrado unificar los mismos, a pesar del esfuerzo por caracterizar de manera más apropiada y precisa esta patología. Entre ellos tanto el ATP -IIla como la IDF han generado definiciones con el objetivo de homogenizar los criterios existentes y salvaguardar el valor del diagnóstico del mismo como marcador de riesgo cardiovascu- 
lar en los pacientes que la presentan. L.M. Sarmiento Méndez et al. (2008) $)^{(15)}$ realizo en España un estudio comparativo entre ambos criterios concluyendo que hace falta muchos más estudios para valorar cual es el criterio más apropiado para prevenir los efectos nocivos de esta enfermedad.

La clasificación de ATP-IIla permite el análisis de todos los criterios del síndrome metabólico, ya que para diagnosticarlo el paciente debe cumplir con 3 de los 5 criterios, ${ }^{(8)}$ en tanto que la IDF define el perímetro abdominal como criterio esencial basándose en el riesgo que ejerce la grasa visceral en la resistencia a la insulina deduciéndole de esta forma valor a los otros criterios diagnósticos y este como principal, sumándole dos o más criterios adicionales. ${ }^{(10)}$ El parámetro de $80 \mathrm{~cm}$ utilizado para definir la obesidad en mujeres excluye a otras personas que podrían tener obesidad según índice de masa corporal (IMC) y que no presenten precisamente $80 \mathrm{~cm}$ o más de perímetro abdominal, al igual en los criterios utilizados por IDF este parámetro no permite incluir en el diagnóstico a aquellas personas que no presentan este perímetro abdominal establecido, pero que presentan otros criterios diagnósticos para síndrome metabólico, excluyéndose a estos individuos en riesgo cardiovascular.

Estas dos definiciones poseen diferencias importantes. En este estudio se revelo las diferencias cuantitativas de las mismas como el incremento en la frecuencia del síndrome metabólico de $55.9 \%$ con los criterios según IDF a $86.3 \%$ utilizando los criterios de ATP-III, sin embargo no hay diferencias estadísticamente significativas al analizar ambos criterios diagnósticos, p>0.05 (Tabla No. 2), comparando estos resultados con los obtenidos en otros estudios observamos que los porcentajes de frecuencia del síndrome metabólico en nuestro país han ido en aumento, aunque no fueron realizados solamente en pacientes diabéticas femeninas, sin embargo nos ayudan a visualizar el panorama general de este conjunto de patologías en el país; en el estudio realizado en El Progreso Yoro, en pacientes hipertensos, se presentó una frecuencia de $70.5 \%$ según ATPIIla, ${ }^{(11)}$ en el estudio realizado en el Hospital Escuela de Tegucigalpa aplicado a pacientes diabéticos e hipertensos se obtuvo frecuencia de $98 \%$ para los diabéticos-hipertensos, $60 \%$ en los diabéticos y $62.5 \%$ en los hipertensos según los criterios del ATP III. ${ }^{12)}$ Cifra que es inferior a los resultados de este estudio donde según la ATP-Illa se obtuvo que $86.3 \%$ de las pacientes tenían síndrome metabólico, siendo todas las pacientes diabéticas.

Al considerar la edad respecto al síndrome metabólico la frecuencia fue mayor en las pacientes de 45 a 59 años de edad con 39.8\% según ATP-IIla y más -frecuente en pacientes mayores de 60 años con un $47.4 \%$ al aplicar IDF. Esto puede ser resultado no solo de edad si no a la sumatoria de todos los factores de riesgo que se enlazan a estas edades de la vida, agravado por el tiempo de persistencia de los síntomas, además de los cambios hormonales y metabólicos que se presentan en las mujeres en esta etapa. Son pocos los estudios que tienen en cuenta a la población femenina y los diversos cambios que se presentan en las etapas de la vida (edad adulta, climaterio y postmenopausia).

Es de interés analizar la frecuencia del síndrome metabólico según el área estudiada. No se encontró diferencia significativa entre área geográfica y la definición de IDF, $p>0.05$ (0.987), sin embargo con los criterios ATP-Illa si se evidencio diferencia significativa entre ambas variables $p<0.05$ (0.017). Probablemente porque IDF basa sus criterios diagnósticos en la obesidad, la cual podría tener mayor frecuencia en determinadas áreas del país por el estilo de vida de cada zona. Para ambos criterios se obtuvo mayor frecuencia de síndrome metabólico en el departamento Santa Bárbara que es un área rural, lo que significa que no se están realizando adecuados programas organizados de prevención de factores de riesgo y promoción de hábitos 
saludables, tanto en ámbitos regionales como nacionales.

Para determinar la frecuencia del síndrome metabólico es importante obtener datos estadísticos sobre esta enfermedad, además de conocer el panorama de la población actual frente al mismo, para constituir programas de prevención que promuevan los cambios terapéuticos y en el estilo de vida, de indudable utilidad para cada una de las patologías que hacen parte del síndrome metabólico.

En conclusión, el síndrome metabólico presenta mayor frecuencia cuando se aplican los criterios según ATP-III en comparación con los de IDF, probablemente debido a que el ATP-III utiliza criterios diagnósticos menos estrictos que IDF. Como se demostró en este estudio no existe correlación estadística entre ambos criterios, por lo que es imprescindible unificar dichos criterios que sean mundial mente aplicables a la población, ya que se dificulta poder comparar la frecuencia del mismo en diferentes países, y no se puede obtener una visión generalizada de la situación actual respecto a esta patología.

\section{AGRADECIMIENTO}

El presente trabajo de investigación fue realizado bajo la supervisión del Dr. Herrera Paz EF a quien expresamos nuestro agradecimiento en la realización del estudio, así también agradecer la colaboración de los pacientes que participaron en el estudio, por su paciencia y tiempo, gracias por su apoyo.

\section{REFERENCIAS BIBLIOGRÁFICAS}

1. Zimmet $P$, Magliano D, Matsuzawa $Y$, Alberti G, Shaw J. The metabolic syndrome: a global public health problem and a new definition. $J$ Atheroscler Thromb. 2005;12(6):295-300.

2. Pinzón JB, Serrano NC, Díaz LA, Mantilla G, Velasco HM, Martínez LX, et al. Impacto de las nuevas definiciones en la prevalencia del síndrome metabólico en una población adulta de Bucaramanga, Colombia. Biomédica. 2007;27(2):172.

Rodríguez Bernardino A, García Polavieja $P$, Jesús Reviriego Fernández, Manuel Serrano Ríos. Prevalencia del síndrome metabólico y grado de concordancia en su diagnóstico en pacientes con diabetes mellitus tipo 2 en España. Endocrinología y Nutrición. 2010; 57(2):60-70.

4. Yadav D, Mahajan S, Subramanian SK, Bisen PS, Chung CH, Prasad GB.. Prevalence of metabolic syndrome in type 2 diabetes mellitus using NCEP-ATPIII, IDF and $\mathrm{WHO}$ definition and Its agreement in gwalior Chambal Region of Central India. Glob JI Health Sci. 2013;5(6):142-55.

5. Guize L, Pannier B, Thomas F, Bean K, Jégo $B$, Benetos $A$. Recent advances in metabolic syndrome and cardiovascular disease. Arch Cardiovasc Dis. 2008; 101(9):577-83.

6. Crepaldi G, Maggi S. El síndrome metabólico: contexto historico. Diabetes veice. 2006;51: 9-10

7. Alberti KG, Zimmet PZ. Definition, diagnosis and classification of diabetes mellitus and its complications. Part 1: diagnosis and classification of diabetes mellitus provisional report of a WHO consultation. Diabet Med. 1998;15(7):539-53.

8. Third Report of the National Cholesterol Education Program (NCEP) Expert Panel on Detection, Evaluation, and Treatment of High Blood Cholesterol in Adults (Adult 
Treatment Panel III) final report. Circulation. 2002;106(25):3143-421.

9. Balkau B, Charles M-A, Drivsholm T, Borch-Johnsen K, Wareham N, Yudkin JS, et al. Frequency of the WHO metabolic syndrome in European cohorts, and an alternative definition of an insulin resistance syndrome. Diabetes Metab. 2002; 28(5):364-76.

10. Alberti KG, Zimmet P, Shaw J. Metabolic syndrome--a new world-wide definition. A Consensus Statement from the International Diabetes Federation. Diabet Med. 2006;23(5):469-80.

11. Hall Martínez J, Hall Reyes J, Alvarenga Thiebaud M, Gómez O. Prevalencia de hipertension en adultos de El Progreso, Yoro, Honduras. Rev Med Hondur. 2005; 73(2):60-4.
12. Barrientos-meja JA, Varela C. Síndrome Metabólico en pacientes diabéticos e hipertensos en la consulta Externa de Medicina Interna. Rev medica postgrados Med UNAH. 2007;10(3):215-20.

13. Kassi E, Pervanidou P, Kaltsas G, Chrousos $G$. Metabolic syndrome: definitions and controversies. BMC Med. 2011; 9(1): 48.

14. Organización Mundial de la Salud. Manual de vigilancia STEPS de la OMS: el método STEPwise de la OMS para la vigilancia de los factores de riesgo de las enfermedades crónicas. Ginebra: World Health Organization; 2006.

15. Sarmiento Méndez LM, Roca-Cusachs Coll A, Arroyo Díaz JA, Benet Gustà MT, Solé Villa MJ, Franco Peral M. Comparison of the definitions of the metabolic syndrome according to ATP III and IDF. Rev Clin Esp 2008; 208(7): 333-8. 